\title{
RNA SARS-CoV-2 Persistence in the Lung of Severe COVID-19 Patients: A Case Series of Autopsies
}

\section{OPEN ACCESS}

Edited by: Leland Shapiro,

University of Colorado Anschutz Medical Campus, United States

Reviewed by:

Martha Ann Delaney,

University of Illinois at Urbana-Champaign, United States Jose Luiz Proenca-Modena,

State University of Campinas, Brazil

*Correspondence: José Palacios

jose.palacios@salud.madrid.org

tThese authors have contributed equally to this work

¥These authors share first authorship

Specialty section: This article was submitted to Infectious Agents and Disease, a section of the journal

Frontiers in Microbiology

Received: 29 November 2021 Accepted: 03 January 2022

Published: 31 January 2022

Citation:

Caniego-Casas T, Martínez-García L, Alonso-Riaño M,

Pizarro D, Carretero-Barrio I,

Martínez-de-Castro N,

Ruz-Caracuel I, de Pablo R, Saiz A,

Royo RN, Santiago A, Rosas M, Rodríguez-Peralto JL, Pérez-Mies $B$, Galán JC and Palacios J (2022) RNA SARS-CoV-2 Persistence in the Lung of Severe COVID-19 Patients: A Case

Series of Autopsies.

Front. Microbiol. 13:824967. doi: 10.3389/fmicb.2022.824967
Tamara Caniego-Casas 1,2,3‡, Laura Martínez-García ${ }^{2,4,5 \neq}$, Marina Alonso-Riaño $6,7,8$, David Pizarro ${ }^{1,2,3}$, Irene Carretero-Barrio ${ }^{1,9}$, Nilda Martínez-de-Castro ${ }^{10}$, Ignacio Ruz-Caracuel' ${ }^{\text {, Raúl de Pablo }}$ 2,9,11, Ana Saiz ${ }^{1,9}$, Rosa Nieto Royo ${ }^{12}$, Ana Santiago1, Marta Rosas, 1,2, José L. Rodríguez-Peralto ${ }^{6,7,8}$, Belén Pérez-Mies 1,2,3,9†, Juan C. Galán ${ }^{2,4,5+}$ and José Palacios ${ }^{1,2,3,9 * \dagger}$

${ }^{1}$ Pathology Department, Hospital Universitario Ramón y Cajal, Madrid, Spain, ${ }^{2}$ Instituto Ramón y Cajal for Health Research (IRYCIS), Madrid, Spain, ${ }^{3}$ CIBERONC, Instituto de Salud Carlos III, Madrid, Spain, ${ }^{4}$ Microbiology Department, Hospital Universitario Ramón y Cajal, Madrid, Spain, ${ }^{5}$ Centro de Investigación Biomédica en Red en Epidemiología y Salud Pública, Madrid, Spain, ${ }^{6}$ Pathology Department, Hospital Universitario 12 de Octubre, Madrid, Spain, ${ }^{7}$ Instituto 12 de Octubre for Health Research, Madrid, Spain, ${ }^{8}$ Faculty of Medicine, Complutense University, Madrid, Spain, ${ }^{9}$ Faculty of Medicine, University of Alcalá, Alcalá de Henares, Spain, ${ }^{10}$ Anaesthesiology and Surgical Critical Care Department, Hospital Universitario Ramón y Cajal, Madrid, Spain, ${ }^{11}$ Medical Intensive Care Unit, Hospital Universitario Ramón y Cajal, Madrid, Spain, ${ }^{12}$ Respiratory Department, Hospital Universitario Ramón y Cajal, Madrid, Spain

The exact role of viral replication in patients with severe COVID-19 has not been extensively studied, and it has only been possible to demonstrate the presence of replicative virus for more than 3 months in a few cases using different techniques. Our objective was to study the presence of RNA SARS-CoV-2 in autopsy samples of patients who died from COVID-19 long after the onset of symptoms. Secondary superimposed pulmonary infections present in these patients were also studied. We present an autopsy series of 27 COVID-19 patients with long disease duration, where pulmonary and extrapulmonary samples were obtained. In addition to histopathological analysis, viral genomic RNA (gRNA) and viral subgenomic RNA (sgRNA) were detected using RT-PCR and in situ hybridization, and viral protein was detected using immunohistochemistry. This series includes 26 adults with a median duration of 39 days from onset of symptoms to death (ranging 9-108 days), 92\% of them subjected to immunomodulatory therapy, and an infant patient. We detected gRNA in the lung of all but one patient, including those with longer disease duration. SgRNA was detected in 11 out of 17 patients (64.7\%) with illness duration up to 6 weeks and in 3 out of 9 patients (33.3\%) with more than 6 weeks of disease progression. Viral protein was detected using immunohistochemistry and viral mRNA was detected using in situ hybridization in 3 out of 4 adult patients with illness duration of $<2$ weeks, but in none of the 23 adult patients with an illness duration of $>2$ weeks. A remarkable result was the detection of viral protein, gRNA and sgRNA in the lung cells of the pediatric patient after 95 days of illness. Additional pulmonary infections included: 9 acute bronchopneumonia, 2 aspergillosis, 2 cytomegalovirus, and $1 \mathrm{BK}$ virus infection. These results suggest that in severe COVID-19, SARS-CoV-2 could persist for longer periods than expected, especially in immunocompromised populations, contributing to the persistence of chronic lung lesions. Additional infections contribute to the fatal course of the disease. 


\section{INTRODUCTION}

COVID-19 is described as a disease with a biphasic course and three stages (early infection, pulmonary, and hyperinflammatory stage) (Siddiqi and Mehra, 2020). The initial phase is characterized by intense viral replication and high viral load, followed by a strong and dysregulated inflammatory response. Nonetheless, it is difficult to specify the duration of each phase. Over the first week, in those patients with mild or moderate COVID-19 disease, the viral load decreases; Conversely, in critical patients, high viral loads have been quantified up to 2 weeks using real-time RT-PCR, and detected by immunohistochemistry (IHC) and in situ hybridization (ISH; Schaefer et al., 2020). Several studies showed that viral RNA could be detected using RT-PCR for periods longer than 2 months (Avanzato et al., 2020; Reuken et al., 2021), but the presence of viral RNA and protein was difficult to demonstrate using ISH and IHC, respectively, after 2 weeks (Evert et al., 2021). Most autopsy studies have been performed in patients with $<30$ days of illness; and therefore, the exact duration of viral RNA presence in lung tissue has not been described comprehensively.

In addition to SARS-CoV-2 infection, patients with severe COVID-19, especially the immunocompromised and those with longer ICU stays are prone to develop other pulmonary infections (Evert et al., 2021), including acute bronchopneumonia, reactivation of herpesviruses such as cytomegalovirus (CMV) and fungal infections, whose frequencies vary among different autopsy series.

Here, we study the presence of RNA SARS-CoV-2 after long periods of illness, using a collection of postmortem specimens from lungs and extra-respiratory organs. We detected genomic SARS-CoV-2 RNA (gRNA) and subgenomic RNA (sgRNA) in 27 patients with a median time to death of 39 days. In addition, we evaluated the presence of other pulmonary infections in these patients with severe COVID-19.

\section{MATERIALS AND METHODS}

\section{Autopsies}

The Research Ethics Committee approved the study (reference: Necropsias_Covid19; 355_20). It included all consecutive autopsies of COVID-19 patients performed at Hospital Universitario Ramón y Cajal (Madrid, Spain) from April 2020 to March $2021(n=26)$. The autopsies corresponded to patients with severe respiratory disease and were requested by the medical staff according to clinical interest and represented about $3 \%$ of all COVID-19 deaths during this period. Consequently, the series does not represent the complete spectrum of causes of death attributable to COVID-19. All autopsies were performed under the consent of the patients' relatives and carried out following safety protocols, in a negative pressure autopsy room, using personal protection equipment, as previously reported (The COVID-19 Autopsy Project, 2020). All autopsies were performed in less than $24 \mathrm{~h}$ after the patient's
TABLE 1 | Clinical characteristics of adult patients.

\begin{tabular}{|c|c|c|c|}
\hline $\begin{array}{l}\text { Demographics } \\
\text { and clinical } \\
\text { characteristics }\end{array}$ & & & $\begin{array}{c}\text { Total number of } \\
\text { observations }\end{array}$ \\
\hline \multirow[t]{2}{*}{ Age, years } & Median (IQR) & $69.50(14.75)$ & 26 \\
\hline & Min, max & 52,91 & \\
\hline Gender, $n(\%)$ & Male & $21(80.77)$ & 26 \\
\hline \multirow[t]{2}{*}{ Weight, kg } & Median (IQR) & $80(20)$ & 25 \\
\hline & Min, $\max$ & 53,109 & \\
\hline $\mathrm{DM}, n(\%)$ & & $2(7.69)$ & 26 \\
\hline Hypertension, n (\%) & & $11(42.30)$ & 26 \\
\hline $\begin{array}{l}\text { Patients admitted } \\
\text { to ICU, } n(\%)\end{array}$ & & 22 (84.61) & 26 \\
\hline \multirow[t]{2}{*}{ Total days } & Median (IQR) & $39(13.25)$ & 26 \\
\hline & Min, $\max$ & 9,108 & \\
\hline \multirow[t]{2}{*}{ Hospitalization days } & Median (IQR) & $30.5(11.75)$ & 26 \\
\hline & Min, max & 3,69 & \\
\hline \multirow[t]{2}{*}{ ICU days } & Median (IQR) & $25.50(16)$ & 22 \\
\hline & Min, max & 12,95 & \\
\hline $\begin{array}{l}\text { Mechanical } \\
\text { ventilation, } n(\%)\end{array}$ & & $22(84.62)$ & 26 \\
\hline $\begin{array}{l}\text { Corticosteroids } \\
\text { use, } n(\%)\end{array}$ & & $24(92.31)$ & 26 \\
\hline $\begin{array}{l}\text { Immunomodulatory } \\
\text { therapy* } n(\%)\end{array}$ & & $23(88.46)$ & 26 \\
\hline
\end{tabular}

*Including tocilizumab and/or interferon $\beta 1 a$.

death and included both gross and histologic examination of the organs.

In the first 14 consecutive decedents, because of biosecurity concerns, we took in-corpore representative sections from the heart, lungs, liver, kidney, pancreas, and bone marrow. Due to improved technical training regarding handling of instruments and sampling collection while wearing personal protection equipment, in the rest of the patients we extracted the complete heart and lung block, left kidney, spleen, and sections from the liver, pancreas, and bone marrow. One autopsy was limited to the lungs, as requested under the consent of the patient's relatives.

\section{Histopathological Evaluation}

After fixation in $10 \%$ buffered formalin for 24-48 h, samples from the five pulmonary lobes were taken in all patients. All histological evaluations were blinded to clinical data. The histopathological classification of the diffuse alveolar damage (DAD) lesions was performed according to Li et al. (2021), as previously reported (Pérez-Mies et al., 2021).

In addition to hematoxylin and eosin stain, PAS and Grocott stains were performed in suspicious cases to highlight fungal infections. Immunohistochemistry for Herpesvirus (clone 10A3, Roche), CMV (clone CCH2 + DDG2, Agilent) and polyomavirus BK (anti-SV40, clone Pab416, Gennova) were performed on a Dako Omnis platform and the staining was visualized with the EnVision system FLEX/HRP (Agilent, 


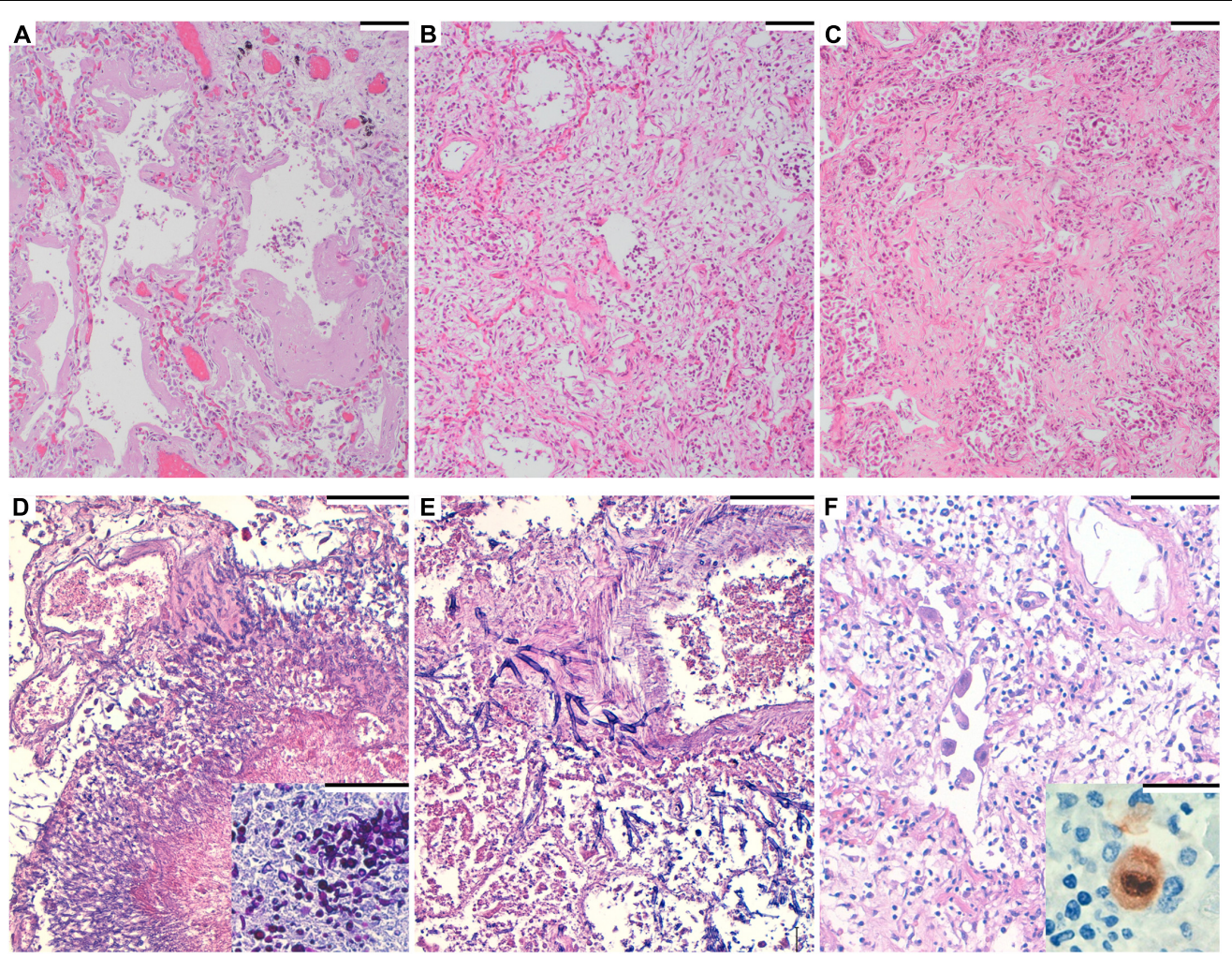

FIGURE 1 | (A) Hematoxylin and eosin. Diffuse alveolar damage (DAD), exudative phase with hyaline membranes formation. Scale bar: 100 m. (B) Hematoxylin and eosin. DAD, organizing phase. Scale bar: $100 \mu \mathrm{m}$. (C) Hematoxylin and eosin. DAD, fibrotic phase. Scale bar: $100 \mu \mathrm{m}$. (D) Hematoxylin and eosin. Numerous filamentous fungi are seen in the lung. Scale bar: $100 \mu \mathrm{m}$. Inset: Periodic acid-Schiff staining, highlighting the fungi. (E) Hematoxylin and eosin. Filamentous fungi invading the arteriolar wall in angioinvasive aspergillosis. Septate hyphae with dichotomous branching at acute angles of around $45^{\circ}$ are apparent. Scale bar: $100 \mu \mathrm{m}$. (F) Hematoxylin and eosin. Endothelial pulmonary cells with viral cytopathic changes. Scale bar: $100 \mu \mathrm{m}$. Inset: Immunohistochemistry for cytomegalovirus, showing positive staining.

TABLE 2 | Lung pathological findings.

\begin{tabular}{|c|c|c|c|}
\hline & & & $\begin{array}{c}\text { Total number of } \\
\text { observations }\end{array}$ \\
\hline \multirow{4}{*}{$\begin{array}{l}\text { Patients with } \\
\text { predominant } \\
\text { pattern, } n(\%)\end{array}$} & Normal lung & $1(3.85)$ & 26 \\
\hline & Exudative DAD & $6(23.07)$ & 26 \\
\hline & $\begin{array}{l}\text { Proliferative/organizing } \\
\text { DAD }\end{array}$ & $15(57.69)$ & 26 \\
\hline & Fibrotic DAD & $4(15.38)$ & 26 \\
\hline $\begin{array}{l}\text { Vascular thrombi, } n \\
\text { (\%) }\end{array}$ & & 18 (69.23) & 26 \\
\hline Endothelialitis, $n$ (\%) & & $11(42.31)$ & 26 \\
\hline \multicolumn{4}{|l|}{ Infections, $n(\%)$} \\
\hline & $\begin{array}{l}\text { Acute } \\
\text { bronchopneumonia }\end{array}$ & $11(42.31)$ & 26 \\
\hline & Aspergillosis & $2(7.69)$ & 26 \\
\hline & Cytomegalovirus & $2(7.69)$ & 26 \\
\hline & Subpleural abscess & $1(3.85)$ & 26 \\
\hline
\end{tabular}

Santa Clara, CA, United States). These cases were selected by morphological features in lung samples suspicious of those infections.

\section{SARS-CoV-2 RNA Detection by RT-PCR}

SARS-CoV-2 detection was done in post-mortem swabs taken from the nasopharynx (NPS), lungs (LS) (the two superior lobes), heart, liver, and right kidney. In addition, testing was done in post-mortem formalin-fixed paraffin-embedded (FFPE) tissue from the lungs (all lobes), heart, liver and kidney in all patients, and large intestine in eight patients. RT-PCR was utilized for detection of genomic SARS-CoV-2 RNA (gRNA) and subgenomic viral RNA (sgRNA) in all patients.

For gRNA detection, swab samples were sent on the same day to the Microbiology Department for the detection of genomic SARS-CoV-2 RNA. RNA extraction and RT-PCR amplification were performed within $3 \mathrm{~h}$ after reception in the laboratory. RNA extraction was performed using MagmaxTM Core Nucleic Acid Purification Kit (Thermo Fisher, Waltham, MA, United States) and gRNA SARS-CoV-2 was detected using TaqmanTM 2019 nCoV assay (Thermo Fisher, Waltham, MA, United States). Samples with a cycle threshold (Ct) lower than 40 were considered positive.

For FFPE samples, RNA was extracted from 10 sections of $5 \mu \mathrm{m}$ obtained from paraffin blocks using RecoverAll Total Nucleic Acid Isolation Kit (Invitrogen), following the manufacturer's instructions. RNA quantity was measured 
TABLE 3 | Features of patients with a cytomegalovirus (CMV) or aspergillosis diagnosis.

\begin{tabular}{|c|c|c|c|c|}
\hline & Patient 6 & Patient 7 & Patient 8 & Patient 12 \\
\hline Age (years) & 60 & 68 & 72 & 55 \\
\hline Pathological CMV and/or aspergillosis diagnosis & CMV & Aspergillosis & CMV & Aspergillosis \\
\hline Clinical CMV and/or aspergillosis diagnosis & No & Aspergillus flavus & CMV & No \\
\hline Illness duration (days) & 32 & 32 & 34 & 37 \\
\hline Hospitalization duration (days) & 29 & 18 & 26 & 30 \\
\hline UCl stay (days) & 19 & 12 & 25 & 30 \\
\hline Comorbidities & None & $\begin{array}{l}\text { Oropharyngeal squamous cell } \\
\text { carcinoma, former smoker, } \\
\text { stroke }\end{array}$ & $\begin{array}{l}\text { Bladder carcinoma, chronic } \\
\text { heart disease }\end{array}$ & None \\
\hline Corticosteroids use & Yes & Yes & Yes & Yes \\
\hline Immunomodulatory therapy (Tozilizumab) & Yes & Yes & Yes & Yes \\
\hline
\end{tabular}

ICU, intensive care unit; CMV, cytomegalovirus.

fluorometrically with Qubit RNA high-sensitivity assay kit (Invitrogen, Waltham, MA, United States). gRNA SARS-CoV-2 was detected in the same way as the swab samples, using TaqmanTM $2019 \mathrm{nCoV}$ assay (Thermo Fisher, Waltham, MA, United States). Samples with a Ct lower than 40 were considered positive.

sgRNA was detected using RT-PCR. Retrotranscription was performed with High-Capacity cDNA Reverse Transcription Kits (Thermo Fisher Scientific), following the manufacturer's instructions. Specific primers were designed between the leader region and the nucleocapsid protein $(\mathrm{N})$ gene (forward primer sequence: $5^{\prime}$ ACCTTCCCAGGTAACAAACCA $3^{\prime}$; reverse primer sequence: 5' GGTCCACCAAACGTAATGCG $3^{\prime}$; amplicon size $129 \mathrm{bp}$ ). The PCR was performed at $54^{\circ}$ annealing temperature for 45 cycles. PCR products were run with High Sensitivity D1000 ScreenTape kit on the Tape Station 2200 (Agilent, Santa Clara, CA, United States). Confirmation of positive fragments was performed using Sanger sequencing.

As internal quality controls, RNAseP or MS2 were used. Only those PCR assays yielding a positive amplification for internal controls were validated following the manufacture's guidelines.

\section{SARS-CoV-2 Immunohistochemistry and in situ Hybridization}

In each patient, the FFPE RT-PCR positive lung sample with the lowest $\mathrm{Ct}$ value was also analyzed with IHC and ISH.

For SARS-CoV-2 immunohistochemistry, we used the monoclonal antibody (1A9, Genetex Inc., Irvine CA, United States) against the spike protein of SARS-Cov/SARSCoV-2 in 1:100 dilution on $3 \mu \mathrm{m}$ slides obtained from paraffin blocks. Antigen retrieval was performed with $10 \mathrm{mM}$ citrate buffer ( $\mathrm{pH}$ 6.0). The staining was visualized with the EnVision system FLEX/HRP (Agilent, Santa Clara, CA, United States).

RNA ISH for SARS-CoV-2 was performed using the RNAscope $^{\circledR}$ SARS-CoV-2 probes for the SARS-CoV-2 S gene encoding the spike protein (catalog \#848561, Advanced Cell Diagnostics, Inc., Hayward, CA, United States) on a Leica Bond III automated stainer (Leica Biosystems, Wetzlar, Germany $\iota$ ), according to the manufacturer's instructions. Briefly, $4-\mu \mathrm{m}$ formalin-fixed and paraffin-embedded tissue sections were
TABLE 4 | Main pathological findings in other organs.

\begin{tabular}{|c|c|c|}
\hline & & $\begin{array}{c}\text { Total number of } \\
\text { observations }\end{array}$ \\
\hline \multicolumn{3}{|l|}{ Heart } \\
\hline Coronary artery atherosclerosis, $n(\%)$ & $4(16)$ & 25 \\
\hline Left ventricle hypertrophy, $n(\%)$ & $3(12)$ & 25 \\
\hline Chronic epicardial inflammation, $n$ (\%) & $1(4)$ & 25 \\
\hline Myocarditis, $n(\%)$ & $1(4)$ & 25 \\
\hline Senile amyloidosis, $n$ (\%) & $1(4)$ & 25 \\
\hline \multicolumn{3}{|l|}{ Liver } \\
\hline Centrolobulillar necrosis, $n$ (\%) & $4(16)$ & 25 \\
\hline Esteatosis, $n(\%)$ & $7(28)$ & 25 \\
\hline Cirrhosis, $n(\%)$ & $1(4)$ & 25 \\
\hline \multicolumn{3}{|l|}{ Kidney } \\
\hline Ischemic necrosis, $n(\%)$ & $8(32)$ & 25 \\
\hline Acute tubular necrosis, $n(\%)$ & $8(32)$ & 25 \\
\hline \multicolumn{3}{|l|}{ Bone marrow } \\
\hline Haemophagocitosys, $n$ (\%) & $19(76)$ & 25 \\
\hline Hyperplasia, $n$ (\%) & $22(88)$ & 25 \\
\hline
\end{tabular}

pre-treated with heat and protease prior to hybridization. Tissue sections were hybridized separately with the target probe to detect infected cells and with the positive and negative control probes. Specific staining signals were identified as brown, punctate dots present in the cytoplasm.

\section{Ultrastructural Study}

We performed a transmission electron microscopic examination of the FFPE tissue from the lung of the pediatric patient. Thin sections were stained with uranyl acetate and lead citrate and were examined with an EM-10 Zeiss microscope.

\section{RESULTS}

\section{Demographic and Pathological Findings}

This series included 26 adults and 1 pediatric patient. The clinical data of the adult patients are shown in Table 1, including 21 males 

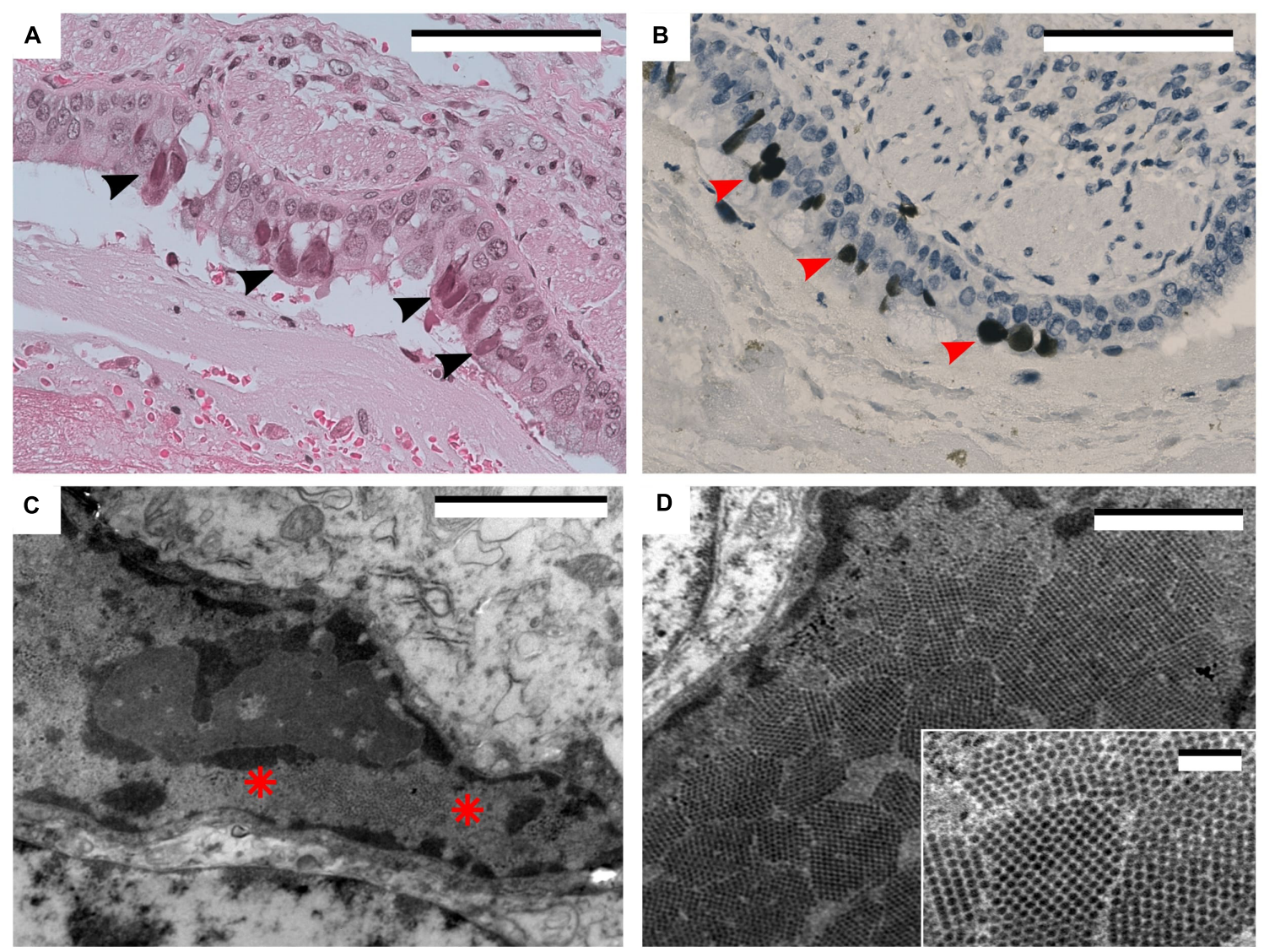

FIGURE 2 | (A) Hematoxylin and eosin. Ciliated bronchial epithelial cells with amphophilic nuclear inclusions (arrowheads). Scale bar: $100 \mu \mathrm{m}$. (B) Polyomavirus BK immunohistochemistry. Same area as panel (A), showing positive staining in the nuclear inclusions (arrowheads). Scale bar: $100 \mu \mathrm{m}$. (C) Electron micrograph of an infected cell containing intranuclear inclusions (between asterisks). Scale bar: $2 \mu \mathrm{m}$. (D) Intranuclear icosahedral inclusions. Scale bar: $1 \mu \mathrm{m}$. Inset: Note the $40 \mathrm{~nm}$ particles characteristic of the polyoma virus group. Scale bar: $200 \mathrm{~nm}$.

and 5 females, with a median age of 69.5 years (IQR 14.8) and a median illness duration of 39 days (IQR 13.3).

Histopathological analysis revealed severe diffuse alveolar damage (DAD) in all but one patient, in whom only occasional areas of hyaline membranes were found. The predominant lung pattern was DAD in fibroproliferative stage, but exudative or fibrotic lesions were also present in different proportions in each patient (Figures 1A-C and Table 2).

Under histological examination, lesions of acute bronchopneumonia were observed in 11 patients (42.3\%). Moreover, we observed the presence of hyphae within areas of mixed inflammatory infiltrates in two patients. These hyphae were visible under the PAS and Grocott techniques (Figures 1D,E) and were also present in the parenchyma of the heart and kidney of one patient. In one case fungi were identified as Aspergillus flavus using PCR (partial sequencing of $18 \mathrm{~S} \mathrm{rDNA}$ ). The fungal structures of the other case were not identified by PCR nor culture. We observed intranuclear and intracytoplasmic inclusions in respiratory epithelial cells, suggestive of CMV infections, in 2 patients (7.7\%). Specific immunohistochemistry for CMV was performed and rendered positive results (Figure 1F). We did no detect CMV inclusions in any other organ. The clinical characteristics of patients with CMV and aspergillosis diagnosis are specified in Table 3.

Other pathological findings in the lungs, hearts, kidneys, livers, and bone marrows are enumerated in Table 4 .

This series also included an 8-year-old boy with primary immunodeficiency (PI). This patient was subjected to a haploidentical transplant due to a severe PI characterized by an almost total absence of naïve $\mathrm{T}$ cells and $\mathrm{T}$ cell receptor excision circles (TRECs). A next generation sequencing study did not identify any pathogenic mutations in the genes related to the main severe combined immunodeficiencies. The patient was waiting for a comparative genomic hybridization study to evaluate the possibility of a 22q11 related syndrome. During his stay in the hospital for the diagnosis of his primary disease, SARS-CoV2 was identified in a NPS during a febrile episode with little respiratory symptoms. The patient recovered from the clinical symptoms but the NPS stayed positive. He developed acute respiratory insufficiency 95 days after the initial diagnosis of COVID-19 and eventually died. In the autopsy, the main pulmonary finding was an extensive bilateral pulmonary hemorrhage. Moreover, some bronchial epithelial cells showed amphophilic nuclear inclusions that 


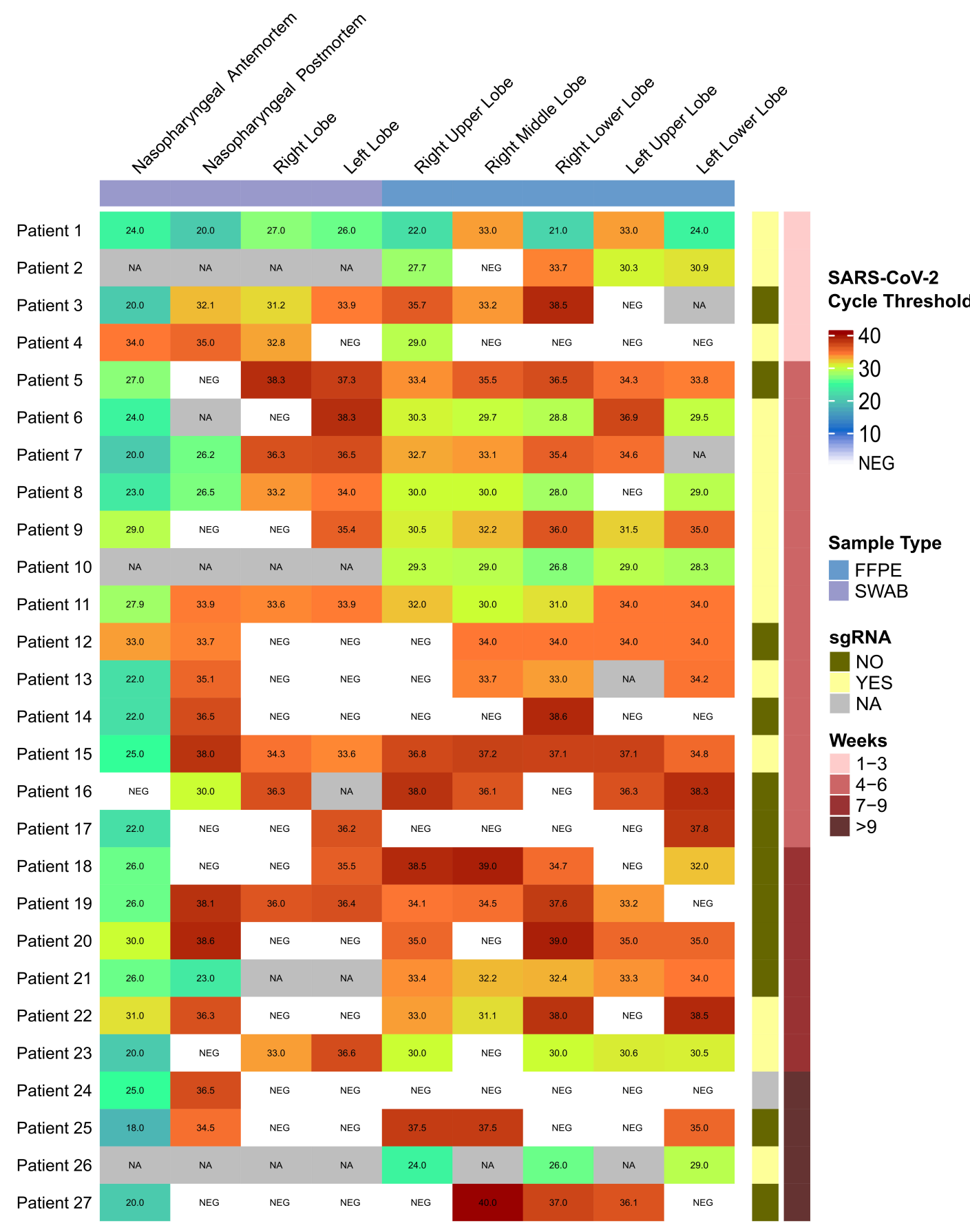

FIGURE 3 | SARS-CoV-2 cycle threshold values heatmap visualization. Upper blue bar indicates sample type (swab or formalin fixed paraffin embedded tissue -FFPE) of nasopharyngeal or lung locations. Right red bar indicates the weeks from onset of symptoms and right yellow bar shows the presence of sub-genomics regions. For antemortem nasopharyngeal swabs, three positive patients did not have the Ct values recorded (NA). One additional patient had a negative result in our hospital, but a positive result in another center. For post-mortem samples, NA indicates no sample available. The infant corresponds to Patient 26.

were positive for Polyomavirus BK immunohistochemistry. Electron microscopic examination revealed multiple intranuclear viral particles arranged in paracrystalline arrays, approximately $40 \mathrm{~nm}$ in diameter, with no envelope, typical of Polyomavirus BK (Figure 2).

\section{SARS-CoV-2 Detection}

All patients tested positive for SARS-CoV-2 in NPS during the development of their disease. Ct values are shown in Figure 3. Three patients who tested positive did not have their $\mathrm{Ct}$ values recorded, and one additional patient had 


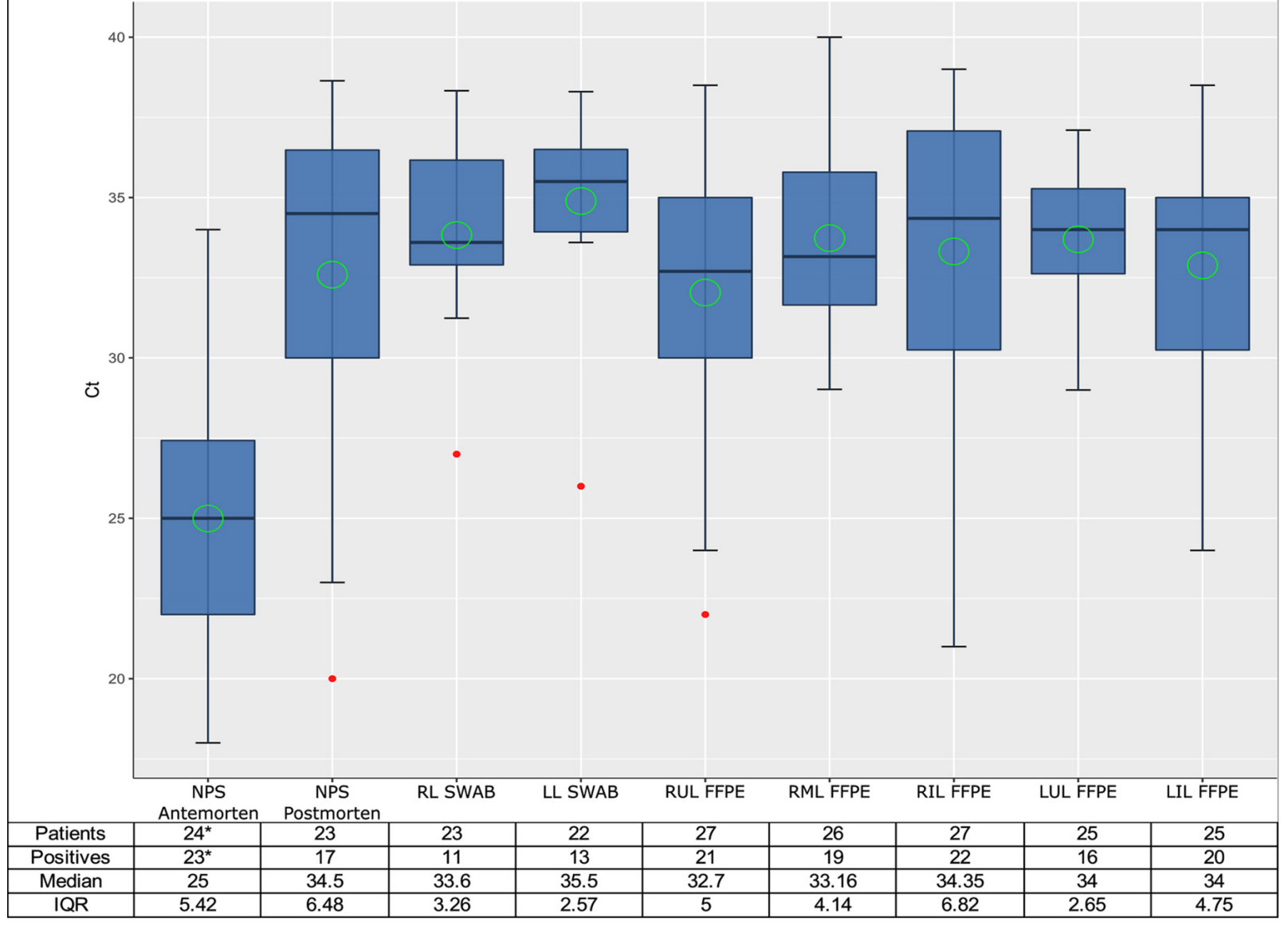

FIGURE 4 | Box plot of SARS-CoV-2 cycle threshold values of the different types of samples. Median is shown as a black line, mean as a green circle and the outliers as red points. ANOVA: $\operatorname{Pr}(>\mathrm{F})=4.04 \mathrm{e}-12^{\star \star \star}$. Ct, cycle threshold; NPS, nasopharyngeal; RL, right lobe; LL, left lobe; RUL, right upper lobe; RML, right medium lobe; RIL, right inferior lobe; LUL, left upper lobe; LIL, left inferior lobe; IQR, interquartile range. *Three positive patients did not have the Ct values recorded. One additional patient had a negative result in our hospital, but a positive result in another Center.

a negative result in our hospital, but a positive one in another center.

SARS-CoV-2 gRNA was detected in at least one autopsy sample in all patients (Figure 3). The number of samples obtained in the respiratory tract, the number of positive samples for each location, and the median, mean and range $\mathrm{Ct}$ values for each location are presented in Figure 4, as well as the comparison of Ct values between premortem swabs and post-mortem samples.

Positive post-mortem NPS were obtained in 17 of $23(73.9 \%)$ patients and positive LS were obtained in 15 of 23 (65.2\%) patients. No positive results were obtained in swab samples from the heart, kidney, and liver in all patients except one, who showed a positive result in the heart swab.

Regarding FFPE samples, positive results were obtained in all but one patient from one or more lung lobes. The frequency of positive SARS-CoV-2 RT-PCR was significantly higher in FFPE lung samples (96.3\%) than in post-mortem NPS (73.9\%) and LS (65.2\%) ( $p=0.038$ and $p=0.024$, respectively). No statistically significant differences were observed in the Ct values obtained from post-mortem swabs and FFPE positive samples. Ct values from FFPE samples showed a statistically significant positive correlation with illness duration $(r=0.5 ; p=0.011)$ (Figure 5), indicating lower viral shedding with disease progression.

In adult patients, we detected sgRNA in 11 of $17(64.7 \%)$ patients with illness duration up to 6 weeks. Moreover, sgRNA was also detected in 2 of $9(22.2 \%)$ adult patients with more than 6 weeks of disease progression (Figure 3).

Viral protein - using IHC -, and viral mRNA - using ISH - were detected in 3 out of 4 adult patients with illness duration of less than 14 days, but in none of the 22 patients with an illness duration between 15 and 103 days. Signals were observed in areas with hyaline membranes, in the cytoplasm of pneumocytes, some of them desquamated into the alveolar lumen, and more infrequently in the respiratory epithelium of bronchi or bronchioles. Staining of intraalveolar cells, suggestive of macrophages, was also observed. Staining of endothelial cells was inconclusive in our samples.

We detected viral protein using IHC, viral gRNA using ISH and RT-PCR, and sgRNA in the lung of the pediatric patient after 95 days of the onset of symptoms (Figure 6). We did not observe viral particles suggestive of SARS-CoV2 using electron microscope examination. 


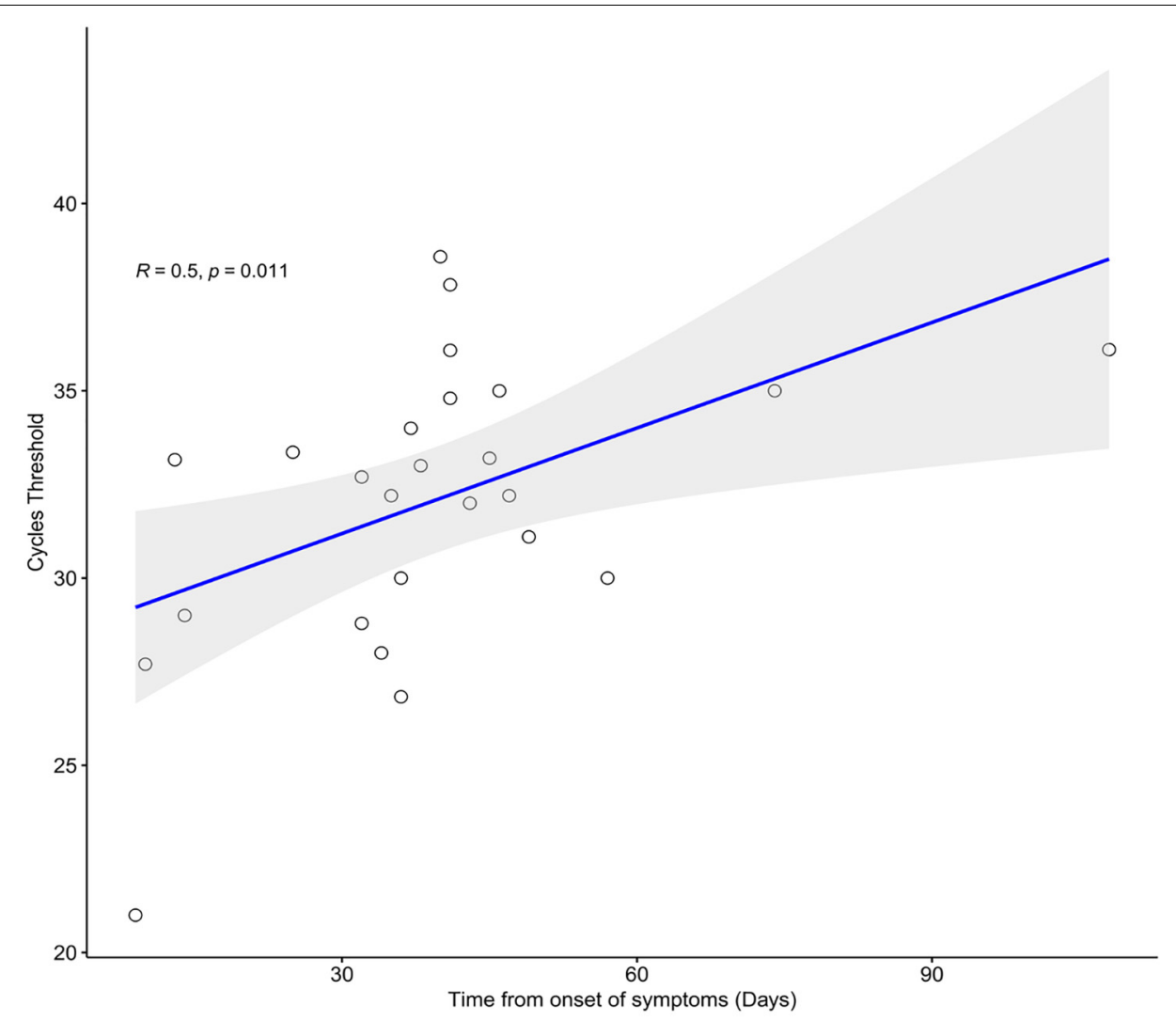

FIGURE 5 | Spearman correlation between days from onset of symptoms and SARS-CoV-2 cycle threshold values of FFPE samples, where regression line is shown in blue and confident interval in gray.

\section{DISCUSSION}

To the best of our knowledge, we report the autopsy series with the longest illness duration in which viral detection has been performed in post-mortem lung samples. The availability of post-mortem specimens has provided a comprehensive insight into the pathophysiology of COVID-19. Several autopsy series including 4 to 64 patients (median 16 patients) with a median duration of illness ranging from 5.7 to 38.5 days (median 14.9 days) have been published (Supplementary Table 1). In this work, we report a 27 -autopsy series with the longest illness duration (median 39 days, ranging 9-108 days) in which viral detection has been performed in post-mortem lung samples, including those with longer disease duration (95 and 108 days). For this reason, this work provides an opportunity to elucidate RNA SARS-CoV-2 persistence in patients with critical illness. According to Supplementary Table 1, only 17 patients in previously reported series showed viral RNA in lung samples after 4 weeks of illness (between 29 and 64 days). The high rate of positivity in our series could be related to the fact that we obtained samples from all lung lobes, a procedure performed in only a few of the previous studies (Supplementary Table 1).

We detected sgRNA in 14 of $26(53.8 \%)$ adult patients, including two who died after 49 and 57 days of disease.
These results are in accordance with the observations of Dorward et al. (2021), who detected sgRNA in a post-mortem lung sample from one patient who died 42 days after the onset of illness. Other studies evaluating sgRNA in autopsy samples were limited to patients with less than 27 days of disease (Desai et al., 2020; Bhatnagar et al., 2021). The role of sgRNA detection as a marker of viral replication is a matter of debate. Whereas different authors have reported sgRNA as a demonstration of persistent viral replication (Santos Bravo et al., 2021), other authors have questioned this claim (Alexandersen et al., 2020; Verma et al., 2021).

We did not detect SARS-Cov-2 mRNA in any organ but the lung in patients with more than 2 weeks of evolution, and no other organs presented lesions related with SARS-Cov-2 infection but the lungs. These facts support the role of persistent lung viral shedding in lung pathology. Moreover, it might suggest a rapid and short extrapulmonary spread of SARS-CoV-2 in the acute phase of the disease, which is generally cleared without triggering any explosive local inflammatory response in other organs (Ferrer-Gómez et al., 2021; Skok et al., 2021).

Persistent RNA viral shedding could be related to host immunity in severe COVID-19 cases, which is supported by the results observed in our pediatric patient with severe primary immunodeficiency. This patient is similar to a few previously reported living patients with secondary immunodeficiency in 

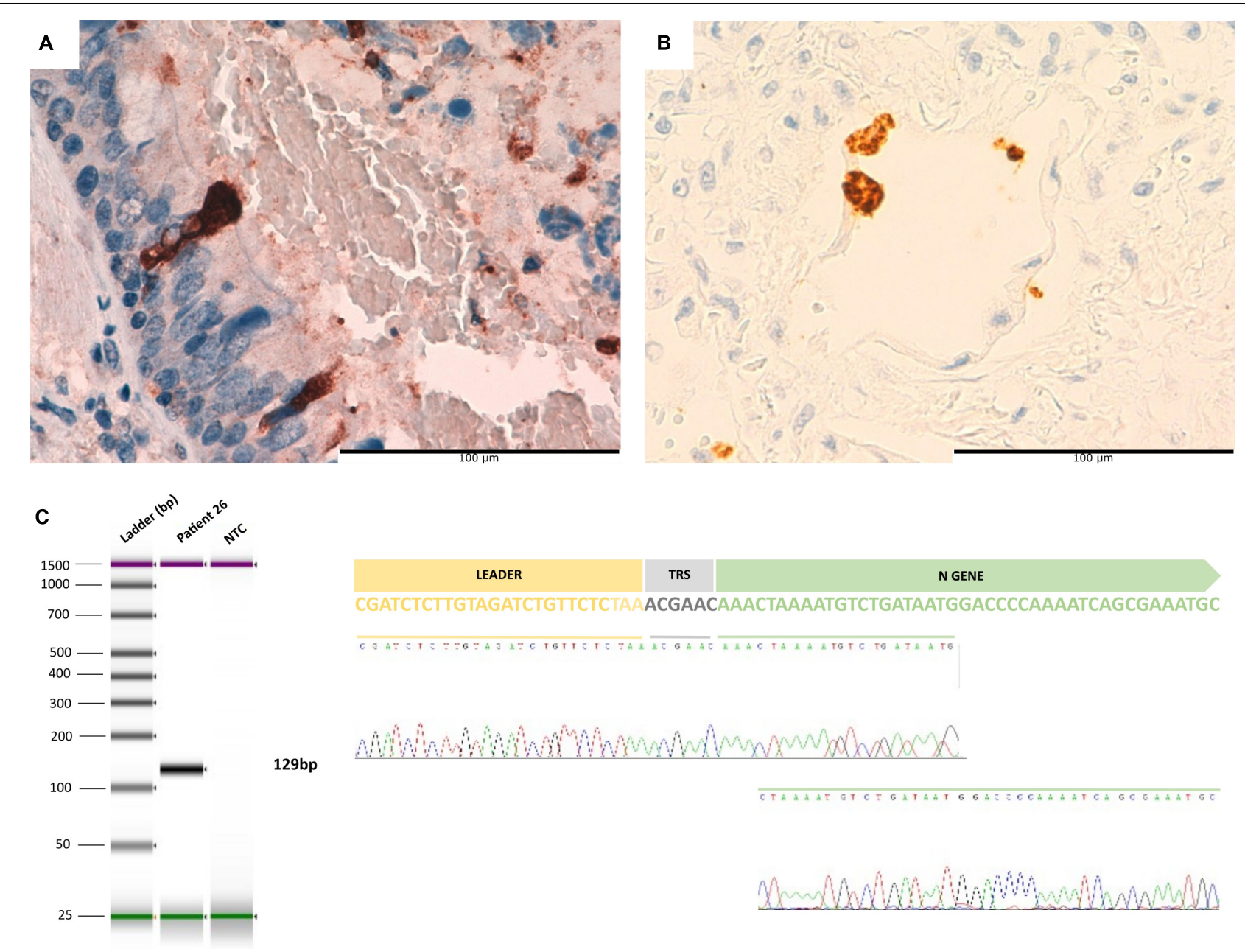

FIGURE 6 | (A) Ciliated bronchial epithelial cells positive for SARS-CoV-2 spike protein in patient 26 (immunohistochemistry). (B) In situ hybridization for SARS-CoV-2 RNA revealed positivity in the cytoplasm of several alveolar cells. (C) From left to right: Tape Station report of sgRNA amplification in infant patient. Electropherogram obtained by Sanger sequencing of the amplified product of gRNA where the leader sequence is observed close to the $\mathrm{N}$ gene.

whom ongoing viral replication was demonstrated for more than 100 days (Avanzato et al., 2020; Baang et al., 2021; Truong et al., 2021). In our case, we were able to demonstrate active viral replication not only by detecting sgRNA with RT-PCR, but also by detecting viral protein and mRNA using IHC and ISH, respectively. As far as we know, this is the only patient reported so far in whom active SARS-CoV-2 viral replication has been demonstrated in the lung after 3 months from the onset of disease. This observation could have important implications for the antiviral treatment of immunocompromised COVID-19 patients with severe lung disease.

The immunocompromised populations, including immunosenescent elderly patients or patients subjected to immunosuppression treatment - which most of the patients in this series were (Table 1), were probably unable to completely eliminate viral particles and the vicious cycle of viral replication and inflammatory response persisted, contributing to chronic deterioration of the lung tissues (Avanzato et al., 2020; Reuken et al., 2021). These observations about viral persistence could have important implications in the selection of new intra-host viral variants (Truong et al., 2021) and consequently the effectiveness of vaccine or antiviral treatment of immunocompromised COVID-19 patients (Sulaiman et al., 2021; Tsukada et al., 2021). The different pandemic waves have been related to the description of new SARS-CoV-2 variants. This raises the question on whether our ISH and IHQ techniques, based on the detection of the spike RNA and protein, respectively, could recognize SARS-CoV-2 variants of concern. However, our study was carried out during the first three pandemic waves, when the SARS-CoV-2 variants B.1, B.1.177 and B.1.1.7 were successively dominant (Hodcroft et al., 2021; López et al., 2021; Martínez-García et al., 2021; Viedma et al., 2021).

Patients hospitalized with COVID-19 have a high risk of secondary infections due to intensive care unit (ICU) admission, prolonged mechanical ventilation, and severe lymphopenia (Ripa et al., 2021). In our series, 13 patients showed secondary pulmonary infections. The frequency of acute 
bronchopneumonia in our series $(42.31 \%)$ was similar to that found in the largest autopsy series published so far, in which the authors found superimposed acute bronchopneumonia in 45 out of 82 patients $(55 \%)$.

It has been suggested that COVID-19-associated pulmonary aspergillosis (CAPA) is a contributing factor to mortality in patients with severe COVID-19 (Salazar et al., 2021). In our series, $7.69 \%$ of the patients showed CAPA. The true incidence of CAPA has not been well established since its diagnosis is challenging from a clinical point of view (Koehler et al., 2021). The frequency of proven CAPA in autopsies varies among series. Thus, among the 24 reviewed series in Supplementary Table 1, only 3 series reported cases of CAPA, with a frequency ranging from 3.8 to $38.1 \%$ (Schurink et al., 2020; Berezowska et al., 2021; Yao et al., 2021). Similarly, whereas (Bryce et al., 2021) reported only 2 patients with CAPA in their series of 100 patients (2\%), (Fortarezza et al., 2021) reported 9 CAPA cases among 45 autopsies (20\%). These authors observed that CAPA increased mainly in the second wave of the pandemic (7 out of 17 vs. 2 out of 28 during the first wave), which was attributed to a higher use of corticosteroids during the second wave.

Corticosteroid use is a known risk factor for CMV reactivation and disease. In our series, 2 patients (7.69\%) showed CMV lung infection. Niitsu et al investigated the frequency and characteristics of CMV infection in 26 critically ill patients with COVID-19 who required mechanical ventilation for more than 1 week (Niitsu et al., 2021). They found that one in four patients developed CMV infection during mechanical ventilation and one patient died from CMV pneumonia. CMV infection was associated with lymphopenia on ICU admission, prolonged mechanical ventilation, and increased mortality. Similar results have been reported in other clinical series (Yamamoto et al., 2021). CMV infection was not reported in any of the 24 autopsy series we reviewed.

In the pediatric patient in this series, we demonstrated the presence of Polyomavirus BK inclusions in epithelial cells using IHC and ultrastructural studies. Pulmonary lesions produced by Polyomavirus BK seem to be very infrequent (Cubukcu-Dimopulo et al., 2000). As far as we know, this is the first case of pulmonary infection by Polyomavirus BK reported in a COVID-19 patient. However, COVID-19 infection has been considered a risk factor for an increasing number of Polyomavirus BK infections in recipients of kidney transplantation (Meshram et al., 2021).

Our study has several limitations. The number of patients is not very high. Moreover, preanalytical issues related with FFPE samples could have negatively affected RNA detection. Finally, we could not test virus infectivity in any sample due to the lack of appropriate biosecure facilities. Despite these limitations, our results suggest a role of viral prolonged presence in the persistence of severe lung lesions in COVID19 patients and of host immunity in the clearance of viral infection, given that immunocompromised patients are more susceptible to prolonged viral infections. Moreover, pulmonary co-infections may have an impact in the prognosis of these patients.

\section{DATA AVAILABILITY STATEMENT}

The raw data supporting the conclusions of this article will be made available by the authors, without undue reservation.

\section{ETHICS STATEMENT}

The studies involving human participants were reviewed and approved by the Research Ethics Committee, Hospital Universitario Ramón y Cajal. Written informed consent to participate in this study was provided by the participants' legal guardian/next of kin. Written informed consent was obtained from the minor(s)' legal guardian/next of kin for the publication of any potentially identifiable images or data included in this article.

\section{AUTHOR CONTRIBUTIONS}

JP, JG, and BP-M contributed to the conception of the study. TC-C, LM-G, ASn, and MR contributed to the sample processing. MA-R, NM-D-C, IR-C, RP, ASi, RR, and JR-P contributed to the data collection. DP, JP, JG, BP-M, TC-C, and LM-G contributed to the data analysis and interpretation. JP, JG, and IC-B drafted the manuscript. All authors revised the manuscript for intellectual content and approved the final version of the manuscript.

\section{FUNDING}

This work was supported by the Instituto de Salud Carlos III (ISCIII) grant PI 19/01331, CIBERONC (grant CB16/12/00316), CIBERESP (grant CB06/02/0053), by the European Development Regional Fund. "A way to achieve Europe" (FEDER), by Merk, Sharp \& Dohme (MSD) and by the Ramón y Cajal Institute for Health Research (IRYCIS) (COVID-19 Grant, 2020/0290). None of the funders participated in the design nor in the manuscript preparation.

\section{ACKNOWLEDGMENTS}

We thank all members of the MACROCOVID and Pathology Departments from Hospital Universitario Ramón y Cajal (Madrid, Spain) for their constant support to the COVID19 Autopsy Project. We also thank the Hospital Universitario Ramón y Cajal-IRyCIS Biobank (Madrid, Spain), for the management of tissue samples.

\section{SUPPLEMENTARY MATERIAL}

The Supplementary Material for this article can be found online at: https://www.frontiersin.org/articles/10.3389/fmicb.2022. 824967/full\#supplementary-material 


\section{REFERENCES}

Alexandersen, S., Chamings, A., and Bhatta, T. R. (2020). SARS-CoV-2 genomic and subgenomic RNAs in diagnostic samples are not an indicator of active replication. Nat. Commun. 11:6059. doi: 10.1038/s41467-020-1 9883-7

Avanzato, V. A., Matson, M. J., Seifert, S. N., Pryce, R., Williamson, B. N., Anzick, S. L., et al. (2020). Case study: prolonged infectious SARS-CoV-2 shedding from an asymptomatic immunocompromised individual with cancer. Cell 183, 1901-1912.e9. doi: 10.1016/j.cell.2020. 10.049

Baang, J. H., Smith, C., Mirabelli, C., Valesano, A. L., Manthei, D. M., Bachman, M. A., et al. (2021). Prolonged severe acute respiratory syndrome coronavirus 2 replication in an immunocompromised patient. J. Infect. Dis. 223, 23-27. doi: 10.1093/infdis/jiaa666

Berezowska, S., Lefort, K., Ioannidou, K., Ndiaye, D.-R., Maison, D., Petrovas, C., et al. (2021). Postmortem cardiopulmonary pathology in patients with COVID-19 infection: single-center report of 12 autopsies from Lausanne, Switzerland. Diagnostics 11:1357. doi: 10.3390/diagnostics110 81357

Bhatnagar, J., Gary, J., Reagan-Steiner, S., Estetter, L. B., Tong, S., Tao, Y., et al. (2021). Evidence of severe acute respiratory syndrome coronavirus 2 replication and tropism in the lungs, airways, and vascular endothelium of patients with fatal coronavirus disease 2019: an autopsy case series. J. Infect. Dis. 223, 752-764. doi: 10.1093/infdis/ jiab039

Bryce, C., Grimes, Z., Pujadas, E., Ahuja, S., Beasley, M. B., Albrecht, R., et al. (2021). Pathophysiology of SARS-CoV-2: the mount sinai COVID-19 autopsy experience. Mod. Pathol. 34, 1456-1467. doi: 10.1038/s41379-02100793-y

Cubukcu-Dimopulo, O., Greco, A., Kumar, A., Karluk, D., Mittal, K., and Jagirdar, J. (2000). BK virus infection in AIDS. Am. J. Surg. Pathol. 24, 145-149. doi: 10.1097/00000478-200001000-00019

Desai, N., Neyaz, A., Szabolcs, A., Shih, A. R., Chen, J. H., Thapar, V., et al. (2020). Temporal and spatial heterogeneity of host response to SARS-CoV2 pulmonary infection. Nat. Commun. 11:6319. doi: 10.1038/s41467-020-2 0139-7

Dorward, D. A., Russell, C. D., Um, I. H., Elshani, M., Armstrong, S. D., PenriceRandal, R., et al. (2021). Tissue-Specific Immunopathology in Fatal COVID19. Am. J. Respir. Crit. Care Med. 203, 192-201. doi: 10.1164/rccm.2020083265OC

Evert, K., Dienemann, T., Brochhausen, C., Lunz, D., Lubnow, M., Ritzka, M., et al. (2021). Autopsy findings after long-term treatment of COVID-19 patients with microbiological correlation. Virchows Arch. 479, 97-108. doi: 10.1007/s00428020-03014-0

Ferrer-Gómez, A., Pian-Arias, H., Carretero-Barrio, I., Navarro-Cantero, A., Pestaña, D., de Pablo, R., et al. (2021). Late cardiac pathology in severe covid19. A postmortem series of 30 patients. Front. Cardiovasc. Med. 8:1372. doi: $10.3389 /$ fcvm. 2021.748396

Fortarezza, F., Boscolo, A., Pezzuto, F., Lunardi, F., Jesús Acosta, M., Giraudo, C., et al. (2021). Proven COVID-19-associated pulmonary aspergillosis in patients with severe respiratory failure. Mycoses 64, 1223-1229. doi: 10.1111/myc. 13342

Hodcroft, E. B., Zuber, M., Nadeau, S., Vaughan, T. G., Crawford, K. H. D., Althaus, C. L., et al. (2021). Spread of a SARS-CoV-2 variant through Europe in the summer of 2020. Nature 595, 707-712. doi: 10.1038/s41586-02103677-y

Koehler, P., Bassetti, M., Chakrabarti, A., Chen, S. C. A., Colombo, A. L., Hoenigl, M., et al. (2021). Defining and managing COVID-19-associated pulmonary aspergillosis: the $2020 \mathrm{ECMM} / \mathrm{ISHAM}$ consensus criteria for research and clinical guidance. Lancet Infect. Dis. 21, e149-e162. doi: 10.1016/S14733099(20)30847-1

Li, Y., Wu, J., Wang, S., Li, X., Zhou, J., Huang, B., et al. (2021). Progression to fibrosing diffuse alveolar damage in a series of 30 minimally invasive autopsies with COVID-19 pneumonia in Wuhan, China. Histopathology 78, 542-555. doi: $10.1111 /$ his. 14249

López, M. G., Chiner-Oms, Á, García de Viedma, D., Ruiz-Rodriguez, P., Bracho, M. A., Cancino-Muñoz, I., et al. (2021). The first wave of the COVID-19 epidemic in Spain was associated with early introductions and fast spread of a dominating genetic variant. Nat. Genet. 53, 1405-1414. doi: 10.1038/s41588021-00936-6

Martínez-García, L., Espinel, M. A., Abreu, M., González-Alba, J. M., Gijón, D., McGee, A., et al. (2021). Emergence and spread of B.1.1.7 lineage in primary care and clinical impact in the morbi-mortality among hospitalized patients in Madrid, Spain. Microorganisms 9:1517. doi: 10.3390/microorganisms 90 71517

Meshram, H. S., Kute, V. B., and Chauhan, S. (2021). BK polyomavirus infection following COVID-19 infection in renal transplant recipients: a singlecenter experience. Kidney Res. Clin. Pract. 40, 496-500. doi: 10.23876/j.krcp. 21.082

Niitsu, T., Shiroyama, T., Hirata, H., Noda, Y., Adachi, Y., Enomoto, T., et al. (2021). Cytomegalovirus infection in critically ill patients with COVID-19. J. Infect. 83, 496-522. doi: 10.1016/j.jinf.2021. 07.004

Pérez-Mies, B., Gómez-Rojo, M., Carretero-Barrio, I., Bardi, T., Benito, A., GarcíaCosío, M., et al. (2021). Pulmonary vascular proliferation in patients with severe COVID-19: an autopsy study. Thorax 76, 1044-1046. doi: 10.1136/thoraxjnl2020-216714

Reuken, P. A., Stallmach, A., Pletz, M. W., Brandt, C., Andreas, N., Hahnfeld, S., et al. (2021). Severe clinical relapse in an immunocompromised host with persistent SARS-CoV-2 infection. Leukemia 35, 920-923. doi: 10.1038/s41375021-01175-8

Ripa, M., Galli, L., Poli, A., Oltolini, C., Spagnuolo, V., Mastrangelo, A., et al. (2021). Secondary infections in patients hospitalized with COVID-19: incidence and predictive factors. Clin. Microbiol. Infect. 27, 451-457. doi: 10.1016/j.cmi. 2020.10 .021

Salazar, F., Bignell, E., Brown, G. D., Cook, P. C., and Warris, A. (2021). Pathogenesis of respiratory viral and fungal coinfections. Clin. Microbiol. Rev. 35:e00094-21. doi: 10.1128/CMR.00094-21

Santos Bravo, M., Nicolás, D., Berengua, C., Fernandez, M., Hurtado, J. C., Tortajada, M., et al. (2021). Severe acute respiratory syndrome coronavirus 2 normalized viral loads and subgenomic RNA detection as tools for improving clinical decision making and work reincorporation. J. Infect. Dis. 224, 13251332. doi: 10.1093/infdis/jiab394

Schaefer, I.-M., Padera, R. F., Solomon, I. H., Kanjilal, S., Hammer, M. M., Hornick, J. L., et al. (2020). In situ detection of SARS-CoV-2 in lungs and airways of patients with COVID-19. Mod. Pathol. 33, 2104-2114. doi: 10.1038/s41379020-0595-z

Schurink, B., Roos, E., Radonic, T., Barbe, E., Bouman, C. S. C., de Boer, H. H., et al. (2020). Viral presence and immunopathology in patients with lethal COVID-19: a prospective autopsy cohort study. Lancet Microbe 1, e290-e299. doi: 10.1016/S2666-5247(20)30144-0

Siddiqi, H. K., and Mehra, M. R. (2020). COVID-19 illness in native and immunosuppressed states: a clinical-therapeutic staging proposal. J. Heart Lung Transplant. 39, 405-407. doi: 10.1016/j.healun.2020. 03.012

Skok, K., Stelzl, E., Trauner, M., Kessler, H. H., and Lax, S. F. (2021). Postmortem viral dynamics and tropism in COVID-19 patients in correlation with organ damage. Virchows Arch. 478, 343-353. doi: 10.1007/s00428-020-0 2903-8

Sulaiman, I., Chung, M., Angel, L., Tsay, J.-C. J., Wu, B. G., Yeung, S. T., et al. (2021). Microbial signatures in the lower airways of mechanically ventilated COVID-19 patients associated with poor clinical outcome. Nat. Microbiol. 6, 1245-1258. doi: 10.1038/s41564-021-0 0961-5

The COVID-19 Autopsy Project (2020). The first COVID-19 autopsy in Spain performed during the early stages of the pandemic. Rev. Española Patol. 53, 182-187. doi: 10.1016/j.patol.2020. 05.004

Truong, T. T., Ryutov, A., Pandey, U., Yee, R., Goldberg, L., Bhojwani, D., et al. (2021). Increased viral variants in children and young adults with impaired humoral immunity and persistent SARS-CoV-2 infection: a consecutive case series. EBioMedicine 67:103355. doi: 10.1016/j.ebiom.2021. 103355

Tsukada, A., Suzuki, M., Kishino, Y., Misumi, K., Igari, T., Nakajima, N., et al. (2021). A kidney transplant patient who died of COVID-19-associated severe 
acute respiratory distress syndrome. Intern. Med. 60, 2297-2300. doi: 10.2169/ internalmedicine.7089-21

Verma, R., Kim, E., Martínez-Colón, G. J., Jagannathan, P., Rustagi, A., Parsonnet, J., et al. (2021). SARS-CoV-2 subgenomic RNA kinetics in longitudinal clinical samples. Open Forum Infect. Dis. 8:ofab310. doi: 10.1093/ofid/ ofab310

Viedma, E., Dahdouh, E., González-Alba, J., González-Bodi, S., Martínez-García, L., Lázaro-Perona, F., et al. (2021). Genomic epidemiology of SARS-CoV2 in Madrid, Spain, during the first wave of the pandemic: fast spread and early dominance by D614G variants. Microorganisms 9:454. doi: 10.3390/ microorganisms 9020454

Yamamoto, Y., Shiroyama, T., Hirata, H., Kuge, T., Matsumoto, K., Yoneda, M., et al. (2021). Prolonged corticosteroid therapy and cytomegalovirus infection in patients with severe COVID-19. J. Med. Virol. 1-7. doi: 10.1002/jmv. 27421 [Epub ahead of print].

Yao, X.-H., Luo, T., Shi, Y., He, Z.-C., Tang, R., Zhang, P.-P., et al. (2021). A cohort autopsy study defines COVID-19 systemic pathogenesis. Cell Res. 31, 836-846. doi: 10.1038/s41422-021-00523-8
Conflict of Interest: The authors declare that the research was conducted in the absence of any commercial or financial relationships that could be construed as a potential conflict of interest.

Publisher's Note: All claims expressed in this article are solely those of the authors and do not necessarily represent those of their affiliated organizations, or those of the publisher, the editors and the reviewers. Any product that may be evaluated in this article, or claim that may be made by its manufacturer, is not guaranteed or endorsed by the publisher.

Copyright (C) 2022 Caniego-Casas, Martínez-García, Alonso-Riaño, Pizarro, Carretero-Barrio, Martínez-de-Castro, Ruz-Caracuel, de Pablo, Saiz, Royo, Santiago, Rosas, Rodríguez-Peralto, Pérez-Mies, Galán and Palacios. This is an open-access article distributed under the terms of the Creative Commons Attribution License (CC BY). The use, distribution or reproduction in other forums is permitted, provided the original author(s) and the copyright owner(s) are credited and that the original publication in this journal is cited, in accordance with accepted academic practice. No use, distribution or reproduction is permitted which does not comply with these terms. 